\title{
On Wolf's regularity criterion of suitable weak solutions to the Navier-Stokes equations
}

\author{
Quanse Jiu * Yanqing Wang ${ }^{\dagger}$ and Daoguo Zhou ${ }^{\ddagger}$
}

\begin{abstract}
In this paper, we consider the local regularity of suitable weak solutions to the 3D incompressible Navier-Stokes equations. By means of the local pressure projection introduced by Wolf in [15, 16], we present a $\varepsilon$-regularity criterion below of suitable weak solutions

$$
\iint_{Q(1)}|u|^{20 / 7} d x d t \leq \varepsilon
$$

which gives an improvement of previous corresponding results obtained in Chae and Wolf [3, Arch. Ration. Mech. Anal., 225: 549-572, 2017], in Guevara and Phuc [6, Calc. Var., 56:68, 2017] and in Wolf [16, Ann. Univ. Ferrara, 61: 149-171, 2015].
\end{abstract}

MSC(2000): 76D03, 76D05, 35B33, 35Q35

Keywords: Navier-Stokes equations; suitable weak solutions; regularity

\section{Introduction}

We focus on the following incompressible Navier-Stokes equations in three-dimensional space

$$
\left\{\begin{array}{l}
u_{t}-\Delta u+u \cdot \nabla u+\nabla \Pi=0, \quad \operatorname{div} u=0, \\
\left.u\right|_{t=0}=u_{0},
\end{array}\right.
$$

where $u$ stands for the flow velocity field, the scalar function $\Pi$ represents the pressure. The initial velocity $u_{0}$ satisfies $\operatorname{div} u_{0}=0$.

In this paper, we are concerned with the regularity of suitable weak solutions to the 3D Navier-Stokes equations (1.1). This kind of weak solutions obeys the local energy inequality below, for a.e. $t \in[-T, 0]$,

$$
\int_{\mathbb{R}^{3}}|u(x, t)|^{2} \phi(x, t) d x+2 \int_{-T}^{t} \int_{\mathbb{R}^{3}}|\nabla u|^{2} \phi d x d s
$$

\footnotetext{
*School of Mathematical Sciences, Capital Normal University, Beijing 100048, PR China. Email: jiuqs@cnu.edu.cn

${ }^{\dagger}$ Department of Mathematics and Information Science, Zhengzhou University of Light Industry, Zhengzhou, Henan 450002, P. R. China Email: wangyanqing20056@gmail.com

${ }^{\ddagger}$ College of Mathematics and Informatics, Henan Polytechnic University, Jiaozuo, Henan 454000, P. R. China Email: zhoudaoguo@gmail.com
} 


$$
\leq \int_{-T}^{t} \int_{\mathbb{R}^{3}}|u|^{2}\left(\partial_{s} \phi+\Delta \phi\right) d x d s+\int_{-T}^{t} \int_{\mathbb{R}^{3}} u \cdot \nabla \phi\left(|u|^{2}+2 \Pi\right) d x d s,
$$

where non-negative function $\phi(x, s) \in C_{0}^{\infty}\left(\mathbb{R}^{3} \times(-T, 0)\right)$.

Before going further, we shall introduce some notations utilized throughout this paper. For $p \in[1, \infty]$, the notation $L^{p}((0, T) ; X)$ stands for the set of measurable functions on the interval $(0, T)$ with values in $X$ and $\|f(t, \cdot)\|_{X}$ belongs to $L^{p}(0, T)$. For simplicity, we write

$$
\|f\|_{L^{p, q}(Q(r))}:=\|f\|_{L^{p}\left(-r^{2}, 0 ; L^{q}(B(r))\right)} \text { and }\|f\|_{L^{p}(Q(r))}:=\|f\|_{L^{p, p}(Q(r))},
$$

where $Q(r)=B(r) \times\left(t-r^{2}, t\right)$ and $B(r)$ denotes the ball of center $x$ and radius $r$.

Roughly speaking, the regularity of suitable weak solutions is intimately connected to $\varepsilon$-regularity criteria (see, e.g., [1, 3, 6, 6, 11, 13 16]). A well-known $\varepsilon$-regularity criterion is the following one with $p=3$ : there is an absolute constant $\varepsilon$ such that, if

$$
\|u\|_{L^{p}(Q(1))}^{p}+\|\Pi\|_{L^{p / 2}(Q(1))}^{p / 2}<\varepsilon
$$

then $u$ is bounded in some neighborhood of point $(0,0)$. This was proved by Lin in $[9]$ (see also Ladyzenskaja and Seregin [8]). In [7], Kukavica proposed three questions regarding this regularity criteria (1.3)

(1) If this result holds for weak solutions which are not suitable.

(2) It is not known if the regularity criteria holds for $p<3$ in (1.3).

(3) If the pressure can be removed from the condition (1.3).

Recently, Guevara and Phuc [6] answered Kukavica's issue (2) via establishing following regularity criteria

$$
\|u\|_{L^{2 p, 2 q}(Q(1))}+\|\Pi\|_{L^{p, q}(Q(1))}<\varepsilon, \quad 3 / q+2 / p=7 / 2 \quad \text { with } 1 \leq q \leq 2 .
$$

Later, He, Wang and Zhou [10] extended Guevara and Phuc's results to

$$
\|u\|_{L^{p, q}(Q(1))}+\|\Pi\|_{L^{1}(Q(1))}<\varepsilon, \quad 1 \leq 2 / p+3 / q<2,1 \leq p, q \leq \infty .
$$

For the question (3), Wolf introduced the local pressure projection (for the detail, see Section 2 ) $\mathcal{W}_{p \Omega}: W^{-1, p}(\Omega) \rightarrow W^{-1, p}(\Omega)(1<p<\infty)$ for a given bounded $C^{2}(\Omega)$ domain $\Omega \subseteq \mathbb{R}^{n}$ in [15, 16] and obtained a $\varepsilon$-regularity criterion below

$$
\iint_{Q(1)}|u|^{3} d x d t<\varepsilon
$$

In addition, very recently, in [3], Wolf and Chae studied Liouville type theorems for selfsimilar solutions to the Navier-Stokes equations by proving $\varepsilon$-regularity criteria

$$
\sup _{-1 \leq t \leq 0} \int_{B(1)}|u|^{q} d x<\varepsilon, \quad \frac{3}{2}<q \leq 3 .
$$

Based Kukavica's questions and recent progresses (1.4)-(1.6), a natural issue is weather the regularity criteria (1.3) holds for $p<3$ without pressure. The goal of this paper is devoted 
to this. Before we state our results, we roughly mention the novelty in [3, 15, 16]. For any ball $B(R) \subseteq \mathbb{R}^{3}$, Wolf et al. introduced the definitions

$$
\nabla \Pi_{h}=-\mathcal{W}_{p, B(R)}(u), \quad \nabla \Pi_{1}=\mathcal{W}_{p, B(R)}(\Delta u), \quad \nabla \Pi_{2}=-\mathcal{W}_{p, B(R)}(u \cdot \nabla u),
$$

and set $v=u+\nabla \Pi_{h}$, then, the local energy inequality reads, for a.e. $t \in[-T, 0]$ and non-negative function $\phi(x, s) \in C_{0}^{\infty}\left(\mathbb{R}^{3} \times(-T, 0)\right)$,

$$
\begin{aligned}
& \int_{B(r)}|v|^{2} \phi(x, t) d x+\int_{-T}^{t} \int_{B(r)}|\nabla v|^{2} \phi(x, t) d x d s \tau \\
\leq & \int_{-T}^{t} \int_{B(r)}|v|^{2}\left(\Delta \phi+\partial_{t} \phi\right) d x d s+\int_{-T}^{t} \int_{B(r)}|v|^{2} u \cdot \nabla \phi d s d s \\
& +\int_{-T}^{t} \int_{B(r)} \phi\left(u \otimes v: \nabla^{2} \Pi_{h}\right) d s d s+\int_{-T}^{t} \int_{B(r)} \phi \Pi_{1} v \cdot \nabla \phi d x d s+\int_{-T}^{t} \int_{B(r)} \phi \Pi_{2} v \cdot \nabla \phi d x d s .
\end{aligned}
$$

It is worth pointing out that any usual suitable weak solutions to the Navier-Stokes system enjoys the local energy inequality (1.8). We refer the reader to [2, Appendix A, p1372] for its proof. As stated in [3, 15, 16], the advantage of local energy inequality (1.8) removed the non-local effect of the pressure term. Based on this, Caccioppoli type inequalities are derived in [3, 15], respectively,

$$
\begin{aligned}
& \|u\|_{L^{3, \frac{18}{5}} Q\left(\frac{1}{2}\right)}^{2}+\|\nabla u\|_{L^{2}\left(Q\left(\frac{1}{2}\right)\right)}^{2} \leq C\|u\|_{L^{3}(Q(1))}^{2}+C\|u\|_{L^{3}(Q(1))}^{3} \cdot \\
& \|u\|_{L^{3, \frac{18}{5}} Q\left(\frac{1}{2}\right)}^{2}+\|\nabla u\|_{L^{2}\left(Q\left(\frac{1}{2}\right)\right)}^{2} \leq C\|u\|_{L^{\frac{3 q}{2 q-3}, q}(Q(1))}^{2}+C\|u\|_{L^{\frac{3 q}{2 q-3}, q}(Q(1))}^{\frac{3 q}{2 q-3}}, \quad \frac{3}{2}<q \leq 3 .
\end{aligned}
$$

Our first result is to derive a new Caccioppoli type inequality

Proposition 1.1. Assume that $u$ is a suitable weak solutions to the Navier-Stokes equations. There holds

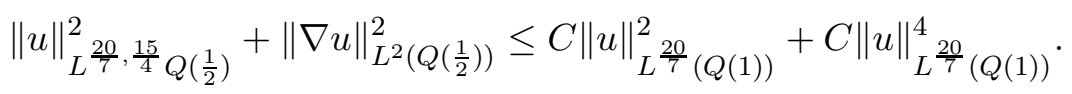

This Caccioppoli type inequality allows us to obtain our main result

Theorem 1.2. Let the pair $(u, \Pi)$ be a suitable weak solution to the 3D Navier-Stokes system (1.1) in $Q(1)$. There exists an absolute positive constant $\varepsilon$ such that if $u$ satisfies

$$
\|u\|_{L^{20 / 7}(Q(1))}<\varepsilon
$$

then, $u \in L^{\infty}(Q(1 / 16))$.

Remark 1.1. This theorem is an improvement of corresponding results in (1.4)-(1.7).

We give some comments on the proof of Proposition 1.1 and Theorem 1.2. Though the non-local pressure disappears in the local energy inequality in (1.8), the velocity field $u$ losses the kinetic energy $\|u\|_{L^{\infty, 2}}$. In contrast with works [3, 15] , owing to $\|u\|_{L^{3, \frac{18}{5}} Q\left(\frac{1}{2}\right)}^{2}$ appearing in Caccioppoli type inequalities in (1.9)-(1.10) and without the kinetic energy of $u$, it seems 
to be difficult to apply the argument used in [3, 6, 10, 15] directly to obtain (1.11). To circumvent these difficulties, first, we observe that every nonlinear term contain at least $v$ in the local energy inequality (1.8). Meanwhile, $v$ enjoys all the energy, namely, $\|v\|_{L^{\infty} L^{2}}$ and $\|v\|_{L^{2} L^{2}}$. It would be natural to absorb $v$ by the left hand of local energy inequality

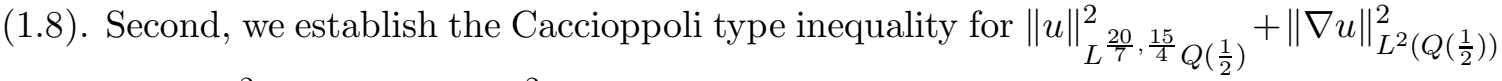

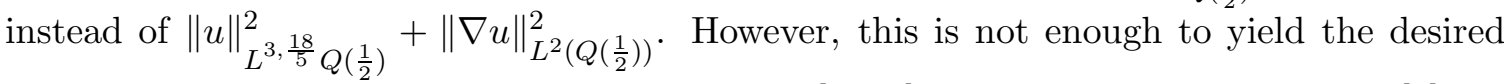
result, which is completely different from that in [6, 10]. To this end, in the spirit of [3], we utilize Caccioppoli type inequality (1.8) and induction arguments developed in [1, 3, 11, 14] to complete the proof of Theorem [1.2. Third, to the knowledge of authors, all previous authors in [1, 13, 11, 14] invoked induction arguments for $\int_{\tilde{Q}_{k}}|v|^{3} \leq \varepsilon_{1}^{2 / 3}$. To bound the term $\iint|v|^{2} \nabla \Pi_{h} \cdot \nabla \phi d \tau$ in local energy inequality (1.8) by $\left.\int_{\tilde{Q}_{k}} v\right|^{3} \leq \varepsilon_{1}^{2 / 3}$, one needs $u \in L^{p}(I ;\|\cdot\|)$ with $p \geq 3$, where $I$ is an time interval. However, from (1.11), we have $u \in L^{p}(I ;\|\cdot\|)$ with $p<3$, therefore, induction arguments with $\iint_{\tilde{Q}_{k}}|v|^{3} \leq \varepsilon_{1}^{2 / 3}$, seems to break down in our case. As said above, since we have all the energy of $v$, we work with

$$
\iint_{\tilde{Q}_{k}}|v|^{\frac{10}{3}} \leq \varepsilon_{1}^{2 / 3}
$$

in induction arguments. Finally, this enables us to achieve the proof of Theorem 1.2 .

The remainder of this paper is structured as follows. In section 2, we explain the detail of Wolf's the local pressure projection $\mathcal{W}_{p, \Omega}$ and present the definition of local suitable weak solutions. Then, we recall some interior estimates of harmonic functions, an interpolation inequality, two classical iteration lemmas and establish an auxiliary lemma utilized in induction arguments. The Caccioppoli type inequality (1.11) is derived in Section 3, Section 4 is devoted to the proof of Theorem 1.2 .

Notations: Throughout this paper, we denote

$$
\begin{array}{lll}
B(x, \mu):=\left\{y \in \mathbb{R}^{n}|| x-y \mid \leq \mu\right\}, & B(\mu):=B(0, \mu), & \tilde{B}(\mu):=B\left(x_{0}, \mu\right), \\
Q(x, t, \mu):=B(x, \mu) \times\left(t-\mu^{2 \alpha}, t\right), & Q(\mu):=Q(0,0, \mu), & \tilde{Q}(\mu):=Q\left(x_{0}, t_{0}, \mu\right), \\
r_{k}=2^{-k}, & \tilde{B}_{k}:=\tilde{B}\left(r_{k}\right), & \tilde{Q}_{k}:=\tilde{Q}\left(r_{k}\right) .
\end{array}
$$

Denote the average of $f$ on the set $\Omega$ by $\bar{f}_{\Omega}$. For convenience, $\bar{f}_{r}$ represents $\bar{f}_{B(r)}$ and $\bar{\Pi}_{\tilde{B}_{k}}$ is denoted by $\tilde{\Pi}_{k}$. $|\Omega|$ represents the Lebesgue measure of the set $\Omega$. We will use the summation convention on repeated indices. $C$ is an absolute constant which may be different from line to line unless otherwise stated in this paper.

\section{Preliminaries}

We begin with Wolf's the local pressure projection $\mathcal{W}_{p, \Omega}: W^{-1, p}(\Omega) \rightarrow W^{-1, p}(\Omega)(1<p<$ $\infty)$. More precisely, for any $f \in W^{-1, p}(\Omega)$, we define $W^{-1, p}(f)=\nabla \Pi$, where $\Pi$ satisfies (2.1). Let $\Omega$ be a bounded domain with $\partial \Omega \in C^{1}$. According to the $L^{p}$ theorem of Stokes system in [4, Theorem 2.1, p149], there exists a unique pair $(u, \Pi) \in W^{1, p}(\Omega) \times L^{p}(\Omega)$ such that

$$
-\Delta u+\nabla \Pi=f, \quad \operatorname{div} u=0,\left.\quad u\right|_{\partial \Omega}=0, \quad \int_{\Omega} \Pi d x=0 .
$$


Moreover, this pair is subject to the inequality

$$
\|u\|_{W^{1, q}(\Omega)}+\|\Pi\|_{L^{q}(\Omega)} \leq C\|f\|_{W^{-1, q}(\Omega)} .
$$

Let $\nabla \Pi=\mathcal{W}_{p, \Omega}(f)\left(f \in L^{p}(\Omega)\right)$, then $\|\Pi\|_{L^{p}(\Omega)} \leq C\|f\|_{L^{p}(\Omega)}$, where we used the fact that $L^{p}(\Omega) \hookrightarrow W^{-1, p}(\Omega)$. Moreover, from $\Delta \Pi=\operatorname{div} f$, we see that $\|\nabla \Pi\|_{L^{p}(\Omega)} \leq C\left(\|f\|_{L^{p}(\Omega)}+\right.$ $\left.\|\Pi\|_{L^{p}(\Omega)}\right) \leq C\|f\|_{L^{p}(\Omega)}$. Now, we present the definition of suitable weak solutions of NavierStokes equations (1.1).

Definition 2.1. A pair $(u, \Pi)$ is called a suitable weak solution to the Navier-Stokes equations (1.1) provided the following conditions are satisfied,

(1) $u \in L^{\infty}\left(-T, 0 ; L^{2}\left(\mathbb{R}^{3}\right)\right) \cap L^{2}\left(-T, 0 ; \dot{H}^{1}\left(\mathbb{R}^{3}\right)\right), \Pi \in L^{3 / 2}\left(-T, 0 ; L^{3 / 2}\left(\mathbb{R}^{3}\right)\right)$;

(2) $(u, \Pi)$ solves (1.1) in $\mathbb{R}^{3} \times(-T, 0)$ in the sense of distributions;

(3) The local energy inequality (1.8) is valid. In addition, $\nabla \Pi_{h}, \nabla \Pi_{1}$ and $\nabla \Pi_{2}$ meet the following fact

$$
\begin{aligned}
& \left\|\nabla \Pi_{h}\right\|_{L^{p}(B(R))} \leq\|u\|_{L^{p}(B(R))}, \\
& \left\|\nabla \Pi_{1}\right\|_{L^{2}(B(R))} \leq\|\nabla u\|_{L^{2}(B(R))}, \\
& \left\|\nabla \Pi_{2}\right\|_{L^{p / 2}(B(R))} \leq\left\|\left.u\right|^{2}\right\|_{L^{p / 2}(B(R))} .
\end{aligned}
$$

We list some interior estimates of harmonic functions $\Delta h=0$, which will be frequently utilized later. Let $1 \leq p, q \leq \infty$ and $p<r<\rho$, then, it holds

$$
\begin{gathered}
\left\|\nabla^{k} h\right\|_{L^{q}(B(r))} \leq \frac{C r^{\frac{n}{q}}}{(\rho-r)^{\frac{n}{p}+k}}\|h\|_{L^{p}(B(\rho))} . \\
\left\|h-\bar{h}_{r}\right\|_{L^{q}(B(r))} \leq \frac{C r^{\frac{n}{q}+1}}{(\rho-r)^{\frac{n}{q}+1}}\left\|h-\bar{h}_{\rho}\right\|_{L^{q}(B(\rho))} .
\end{gathered}
$$

The proof of (2.5) rests on the mean value property of harmonic functions. This together with mean value theorem leads to (2.6). We leave the detail to the reader. For reader's convenience, we recall an interpolation inequality. For each $2 \leq l \leq \infty$ and $2 \leq k \leq 6$ satisfying $\frac{2}{l}+\frac{3}{k}=\frac{3}{2}$, according to the Hölder inequality and the Young inequality, we know that

$$
\begin{aligned}
\|u\|_{L^{k, l}(Q(\mu))} & \leq C\|u\|_{L^{2, \infty}(Q(\mu))}^{1-\frac{2}{l}}\|u\|_{L^{6,2}(Q(\mu))}^{\frac{2}{l}} \\
& \leq C\|u\|_{L^{2, \infty}(Q(\mu))}^{1-\frac{2}{l}}\left(\|u\|_{L^{2, \infty}(Q(\mu))}+\|\nabla u\|_{L^{2}(Q(\mu))}\right)^{\frac{2}{l}} \\
& \leq C\left(\|u\|_{L^{2, \infty}(Q(\mu))}+\|\nabla u\|_{L^{2}(Q(\mu))}\right) .
\end{aligned}
$$

In additon, we recall two well-known iteration lemmas.

Lemma 2.1. [5, Lemma 2.1, p.86] Let $\phi(t)$ be a nonegative and nondecreasing functions on $[0, R]$. Suppose that

$$
\phi(\rho) \leq A\left[\left(\frac{\rho}{r}\right)^{\alpha}+\varepsilon\right] \phi(r)+B r^{\beta}
$$


for any $0<\rho \leq r \leq R$, with $A, B, \alpha, \beta$ nonnegative constants and $\beta<\alpha$. Then for any $\gamma \in(\beta, \alpha)$, there exists a constant $\varepsilon_{0}$ such that if $\varepsilon<\varepsilon_{0}$ we have for all $0<\rho \leq r \leq R$

$$
\phi(\rho) \leq C\left\{\left(\frac{\rho}{r}\right)^{\beta} \phi(r)+B \rho^{\beta}\right\} .
$$

where $c$ is a positive constant depending on $A, \alpha, \beta, \gamma$.

Lemma 2.2. [5, Lemma V.3.1, p.161] Let $I(s)$ be a bounded nonnegative function in the interval $[r, R]$. Assume that for every $\sigma, \rho \in[r, R]$ and $\sigma<\rho$ we have

$$
I(\sigma) \leq A_{1}(\rho-\sigma)^{-\alpha_{1}}+A_{2}(\rho-\sigma)^{-\alpha_{2}}+A_{3}+\ell I(\rho)
$$

for some non-negative constants $A_{1}, A_{2}, A_{3}$, non-negative exponents $\alpha_{1} \geq \alpha_{2}$ and a parameter $\ell \in[0,1)$. Then there holds

$$
I(r) \leq c\left(\alpha_{1}, \ell\right)\left[A_{1}(R-r)^{-\alpha_{1}}+A_{2}(R-r)^{-\alpha_{2}}+A_{3}\right] .
$$

The following lemma is motivated by [3, Lemma 2.9, p.558 ].

Lemma 2.3. Let $f \in L^{q}(Q(1))$ with $q>1$ and $0<r_{0}<1$. Suppose that for all $\left(x_{0}, t_{0}\right) \in$ $Q(1 / 2)$ and $r_{0} \leq r \leq \frac{1}{2}$

$$
\iint_{\tilde{Q}(r)}\left|f-\bar{f}_{\tilde{B}(r)}\right|^{q} \leq C r^{4}
$$

Let $\nabla \Pi=\mathcal{W}_{q, \tilde{B}(1)}(\nabla \cdot f)$. Then for all $\left(x_{0}, t_{0}\right) \in Q(1 / 2)$ and $r_{0} \leq r \leq \frac{1}{4}$, it holds

$$
\iint_{\tilde{Q}(r)}\left|\Pi-\bar{\Pi}_{\tilde{B}(r)}\right|^{q} \leq C r^{4}
$$

Proof. From the definition of pressure projection $\mathcal{W}_{q, B(1)}$, we know that

$$
\|\Pi\|_{L^{q}(B(1))} \leq C\left\|f-\bar{f}_{B(1)}\right\|_{L^{q}(B(1))} .
$$

Let $\phi(x)=1, x \in \tilde{B}\left(\frac{3 r}{4}\right), \phi(x)=0, x \in \tilde{B}^{c}(r)$.

Note that

$$
\Delta \Pi=\operatorname{div} \mathcal{W}_{q, B(1)}^{*}(\nabla \cdot f) .
$$

We set $\Pi=\Pi_{(1)}+\Pi_{(2)}$, where

$$
\Delta \Pi_{(1)}=-\operatorname{div} \mathcal{W}_{q, B(1)}^{*}\left(\nabla \cdot\left[\phi\left(f-\bar{f}_{\tilde{B}(r)}\right)\right]\right),
$$

therefore, as a consequence, it holds

$$
\Delta \Pi_{(2)}=0, x \in \tilde{B}(3 r / 4) .
$$

In view of classical Calderón-Zygmund theorem, we have

$$
\left\|\Pi_{(1)}-\bar{\Pi}_{(1) \tilde{B}(r)}\right\|_{L^{q}(\tilde{B}(r))} \leq C\left\|f-\bar{f}_{\tilde{B}(r)}\right\|_{L^{q}(\tilde{B}(r))} .
$$

Combining this and hypothesis (2.8), we get

$$
\left\|\Pi_{(1)}-\bar{\Pi}_{(1)} \tilde{B}(r)\right\|_{L^{q}(\tilde{Q}(r))} \leq C r^{\frac{4}{q}} .
$$


The interior estimates of harmonic functions (2.6) and the triangle inequality guarantee that, for $\theta<1 / 2$,

$$
\begin{aligned}
& \int_{\tilde{B}(\theta r)} \mid \Pi_{(2)}-\overline{\Pi_{(2)}} \tilde{B}(\theta r) \\
\leq & \frac{C(r \theta)^{q+q} d x}{\left(\frac{r}{2}\right)^{3+q}} \int_{\tilde{B}(r / 2)} \mid \Pi_{(2)}-\overline{\Pi_{(2)}} \tilde{B}(r / 2) \\
\leq & C \theta^{3+q} \int_{\tilde{B}(r / 2)}\left|\Pi-\bar{\Pi}_{\tilde{B}(r / 2)}\right|{ }^{q} d x+\int_{\tilde{B}(r / 2)}\left|\Pi_{(1)}-\bar{\Pi}_{(1)} \tilde{B}(r / 2)\right|{ }^{q} d x .
\end{aligned}
$$

This and (2.10) imply

$$
\left.\iint_{\tilde{Q}(\theta r)}\left|\Pi_{(2)}-\bar{\Pi}_{(2)} \tilde{B}(\theta r)\right|\right|^{q} d x d s \leq C \theta^{3+q} \iint_{\tilde{Q}(r / 2)}\left|\Pi-\bar{\Pi}_{\tilde{B}(r / 2)}\right|^{q} d x d s+C r^{4} .
$$

Utilizing the triangle inequality again, (2.10) and the last inequality, we have

$$
\begin{aligned}
& \iint_{\tilde{Q}(\theta r)}\left|\Pi-\bar{\Pi}_{\tilde{B}(\theta r)}\right|^{q} d x d s \\
& \leq \iint_{\tilde{Q}(\theta r)}\left|\Pi_{(1)}-\bar{\Pi}_{(1)} \tilde{B}(\theta r)\right|{ }^{q} d x d s+\iint_{\tilde{Q}(\theta r)}\left|\Pi_{(2)}-\overline{\Pi_{(2)} \tilde{B}(\theta r)}\right|{ }^{q} d x d s \\
& \leq \iint_{\tilde{Q}(r)}\left|f-\bar{f}_{\tilde{B}(r)}\right|^{q} d x d s+C \theta^{3+q} \iint_{\tilde{Q}(r / 2)}\left|\Pi-\bar{\Pi}_{\tilde{B}(r / 2)}\right|^{q} d x d s+C r^{4} \\
& \leq C \theta^{3+q} \iint_{\tilde{Q}(r)}\left|\Pi-\bar{\Pi}_{\tilde{B}(r)}\right|^{q} d x d s+C r^{4}
\end{aligned}
$$

where we used the fact that $\left\|g-\bar{g}_{B(r)}\right\|_{L^{p}(B(r))} \leq C\|g-c\|_{L^{p}(B(r))}$ with $p \geq 1$.

Now, invoking Lemma 2.1 and (2.9), we see that

$$
\begin{aligned}
\iint_{\tilde{Q}(r)}\left|\Pi-\bar{\Pi}_{\tilde{B}(r)}\right|{ }^{q} d x & \leq C r^{4} \iint_{\tilde{Q}(1 / 4)}\left|\Pi-\bar{\Pi}_{\tilde{B}(1 / 4)}\right|{ }^{q} d x+C r^{4} \\
& \leq C r^{4} \iint_{Q(1)}\left|f-\bar{f}_{B(1)}\right|{ }^{q} d x+C r^{4} \\
& \leq C r^{4} .
\end{aligned}
$$

This completes the proof of this lemma.

\section{Proof of Proposition 1.1}

This section contains the proof of Proposition 1.1. Proposition 1.1 turns out to be a corollary of the following proposition.

Proposition 3.1. Suppose that $(u, \Pi)$ is a suitable weak solution to the Navier-Stokes equations in $Q(R)$. Then there holds, for any $R>0$,

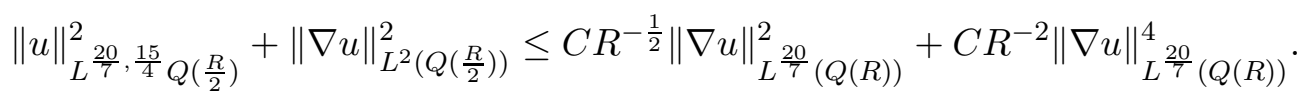


Proof. Consider $0<R / 2 \leq r<\frac{3 r+\rho}{4}<\frac{r+\rho}{2}<\rho \leq R$. Let $\phi(x, t)$ be non-negative smooth function supported in $Q\left(\frac{r+\rho}{2}\right)$ such that $\phi(x, t) \equiv 1$ on $Q\left(\frac{3 r+\rho}{4}\right),|\nabla \phi| \leq C /(\rho-r)$ and $\left|\nabla^{2} \phi\right|+\left|\partial_{t} \phi\right| \leq C /(\rho-r)^{2}$.

Let $\nabla \Pi_{h}=\mathcal{W}_{20 / 7, B(\rho)}(u)$, then, there holds

$$
\begin{aligned}
& \left\|\nabla \Pi_{h}\right\|_{L^{20 / 7}(Q(\rho))} \leq C\|u\|_{L^{20 / 7}(Q(\rho))}, \\
& \left\|\Pi_{1}\right\|_{L^{2}(Q(\rho))} \leq C\|\nabla u\|_{L^{2}(Q(\rho))}, \\
& \left\|\Pi_{2}\right\|_{L^{\frac{10}{7}}(Q(\rho))} \leq C\left\||u|^{2}\right\|_{L^{\frac{10}{7}}(Q(\rho))} .
\end{aligned}
$$

Thanks to $v=u+\nabla \Pi_{h}$, the Hölder inequality and (3.2), we arrive at

$$
\begin{aligned}
\iint_{Q(\rho)}\left|v_{B}\right|^{2}\left|\Delta \phi^{4}+\partial_{t} \phi^{4}\right| & \leq \frac{C}{(\rho-r)^{2}} \iint_{Q\left(\frac{r+\rho}{2}\right)}|u|^{2}+\left|\nabla \Pi_{h}\right|^{2} \\
& \leq \frac{C \rho^{3 / 2}}{(\rho-r)^{2}}\left(\iint_{Q\left(\frac{r+\rho}{2}\right)}|u|^{\frac{20}{7}}+\left|\nabla \Pi_{h}\right|^{\frac{20}{7}}\right)^{\frac{7}{10}} \\
& \leq \frac{C \rho^{3 / 2}}{(\rho-r)^{2}}\left(\iint_{Q(\rho)}|u|^{\frac{20}{7}}\right)^{\frac{7}{10}}
\end{aligned}
$$

Combining Hölder's inequality with interpolation inequality (2.7) and Young's inequality yields

$$
\begin{aligned}
& \iint_{Q(\rho)}|v|^{2} \phi^{3} u \cdot \nabla(\phi) d \tau \\
\leq & \frac{C}{(\rho-r)}\left\|v \phi^{2}\right\|_{L^{10 / 3}\left(Q\left(\frac{r+\rho}{2}\right)\right)}\|v\|_{L^{20 / 7}\left(Q\left(\frac{r+\rho}{2}\right)\right)}\|u\|_{L^{20 / 7}\left(Q\left(\frac{r+\rho}{2}\right)\right)} \\
\leq & \frac{1}{16}\left\|v \phi^{2}\right\|_{L^{10 / 3}\left(Q\left(\frac{r+\rho}{2}\right)\right)}^{2}+\frac{C}{(\rho-r)^{2}}\|v\|_{L^{20 / 7}\left(Q\left(\frac{r+\rho}{2}\right)\right)}^{2}\|u\|_{L^{20 / 7}\left(Q\left(\frac{r+\rho}{2}\right)\right)}^{2} \\
\leq & \frac{1}{16}\left(\left\|v \phi^{2}\right\|_{L^{2, \infty}(Q(\rho))}^{2}+\left\|\nabla\left(v \phi^{2}\right)\right\|_{L^{2}(Q(\rho))}^{2}\right)+\frac{C}{(\rho-r)^{2}}\|u\|_{L^{20 / 7}(Q(\rho))}^{4} .
\end{aligned}
$$

By virtue of interior estimate of harmonic function (2.5) and (3.2), we conclude that

$$
\begin{aligned}
\left\|\nabla^{2} \Pi_{h}\right\|_{L^{20 / 7}\left(Q\left(\frac{r+\rho}{2}\right)\right)} & \leq \frac{(r+\rho)^{\frac{21}{20}}}{(\rho-r)^{\frac{41}{20}}}\left\|\nabla \Pi_{h}\right\|_{L^{20 / 7}(Q(\rho))} \\
& \leq \frac{C \rho^{\frac{21}{20}}}{(\rho-r)^{\frac{41}{20}}}\|u\|_{L^{20 / 7}(Q(\rho))},
\end{aligned}
$$

which leads to

$$
\begin{aligned}
& \iint_{Q(\rho)} \phi^{4}\left(u \otimes v: \nabla^{2} \Pi_{h}\right) \\
\leq & \left\|v \phi^{2}\right\|_{L^{10 / 3}\left(Q\left(\frac{r+\rho}{2}\right)\right)}\|u\|_{L^{20 / 7}\left(Q\left(\frac{r+\rho}{2}\right)\right)}\left\|\nabla^{2} \Pi_{h}\right\|_{L^{20 / 7}\left(Q\left(\frac{r+\rho}{2}\right)\right)} \\
\leq & \frac{1}{16}\left(\left\|v \phi^{2}\right\|_{L^{2, \infty}(Q(\rho))}^{2}+\left\|\nabla\left(\phi^{2} v\right)\right\|_{L^{2}(Q(\rho))}^{2}\right)+\frac{C \rho^{\frac{21}{10}}}{(\rho-r)^{\frac{41}{10}}}\|u\|_{L^{20 / 7}(Q(\rho)}^{4} .
\end{aligned}
$$


In light of Hölder inequality, (3.3) and Young's inequality, we deduce that

$$
\begin{aligned}
\iint_{Q(\rho)} \phi^{3} \Pi_{1} v \cdot \nabla \phi d \tau & \leq \frac{C}{(\rho-r)}\|v\|_{L^{2}\left(Q\left(\frac{r+\rho}{2}\right)\right)}\left\|\Pi_{1}\right\|_{L^{2}\left(Q\left(\frac{r+\rho}{2}\right)\right)} \\
& \leq \frac{C}{(\rho-r)^{2}}\|v\|_{L^{2}\left(Q\left(\frac{r+\rho}{2}\right)\right)}^{2}+\frac{1}{16}\left\|\Pi_{1}\right\|_{L^{2}(Q(\rho))}^{2} \\
& \leq \frac{C \rho^{3 / 2}}{(\rho-r)^{2}}\left(\iint_{Q(\rho)}|u|^{\frac{20}{7}}\right)^{\frac{7}{10}}+\frac{1}{16}\|\nabla u\|_{L^{2}(Q(\rho))}^{2} .
\end{aligned}
$$

We derive from the Hölder inequality, (2.4) and Young's inequality that

$$
\begin{aligned}
\iint_{Q(\rho)} \phi^{3} \Pi_{2} v \cdot \nabla \phi d \tau & \leq \frac{C}{(\rho-r)}\left\|v \phi^{2}\right\|_{L^{\frac{10}{3}}\left(Q\left(\frac{r+\rho}{2}\right)\right)}\left\|\Pi_{2}\right\|_{L^{\frac{10}{7}}}\left(Q\left(\frac{r+\rho}{2}\right)\right) \\
& \leq \frac{1}{16}\|v\|_{L^{\frac{10}{3}}\left(Q\left(\frac{r+\rho}{2}\right)\right)}^{2}+\frac{C}{(\rho-r)^{2}}\left\|\Pi_{2}\right\|_{L^{\frac{10}{7}}(Q(\rho))}^{2} \\
& \leq \frac{1}{16}\left(\left\|v \phi^{2}\right\|_{L^{2, \infty}(Q(\rho))}^{2}+\left\|\nabla\left(\phi^{2} v\right)\right\|_{L^{2}(Q(\rho))}^{2}\right)+\frac{C}{(\rho-r)^{2}}\|u\|_{L^{20 / 7}(Q(\rho)}^{4} .
\end{aligned}
$$

The Cauchy-Schwarz inequality and (3.5) allows us to obtain that

$$
\begin{aligned}
\iint_{Q(\rho)}\left|\nabla\left(v \phi^{2}\right)\right|^{2} d x d s & \leq 2\left(\iint_{Q(\rho)}|\nabla v|^{2} \phi^{4} d x d s+4 \iint_{Q(\rho)}|\nabla \phi|^{2}|v|^{2} \phi^{2} d x d s\right) \\
& \leq 2 \iint_{Q(\rho)}|\nabla v|^{2} \phi^{4} d x d s+\frac{C \rho^{3 / 2}}{(\rho-r)^{2}}\left(\iint_{Q(\rho)}|u|^{\frac{20}{7}}\right)^{\frac{7}{10}} .
\end{aligned}
$$

Substituting (3.5)-(3.9) into (1.8) and using (3.10), we infer that

$$
\begin{aligned}
& \sup _{-\rho^{2} \leq t \leq 0} \int_{B(\rho)}\left|v \phi^{2}\right|^{2} d x+\iint_{Q(\rho)}\left|\nabla\left(v \phi^{2}\right)\right|^{2} d x d \tau \\
\leq & \frac{1}{4}\left(\left\|v \phi^{2}\right\|_{L^{2, \infty}(Q(\rho))}^{2}+\left\|\nabla\left(v \phi^{2}\right)\right\|_{L^{2}(Q(\rho))}^{2}\right)+\left\{\frac{C}{(\rho-r)^{2}}+\frac{C \rho^{\frac{21}{10}}}{(\rho-r)^{\frac{41}{10}}}\right\}\|u\|_{L^{20 / 7}(Q(\rho))}^{4} \\
& +\frac{C \rho^{3 / 2}}{(\rho-r)^{2}}\|u\|_{L^{20 / 7}(Q(\rho))}^{2}+\frac{1}{16}\|\nabla u\|_{L^{2}(Q(\rho))}^{2},
\end{aligned}
$$

that is,

$$
\begin{aligned}
& \sup _{-\rho^{2} \leq t \leq 0} \int_{B(\rho)}\left|v \phi^{2}\right|^{2} d x+\iint_{Q(\rho)}\left|\nabla\left(v \phi^{2}\right)\right|^{2} d x d \tau \\
\leq & \left\{\frac{C}{(\rho-r)^{2}}+\frac{C \rho^{\frac{21}{10}}}{(\rho-r)^{\frac{41}{10}}}\right\}\|u\|_{L^{20 / 7}(Q(\rho))}^{4} \\
& +\frac{C \rho^{3 / 2}}{(\rho-r)^{2}}\|u\|_{L^{20 / 7}(Q(\rho))}^{2}+\frac{1}{16}\|\nabla u\|_{L^{2}(Q(\rho))}^{2},
\end{aligned}
$$

Together with interior estimate of harmonic function (2.5) and (3.2) implies that

$$
\begin{aligned}
\left\|\nabla \Pi_{h}\right\|_{L^{\frac{20}{7}, \frac{15}{4}} Q(r)}^{2} & \leq \frac{C r^{\frac{8}{5}}}{(\rho-r)^{\frac{21}{10}}}\left\|\nabla \Pi_{h}\right\|_{L^{\frac{20}{7}} Q(\rho)}^{2} \\
& \leq \frac{C r^{\frac{8}{5}}}{(\rho-r)^{\frac{21}{10}}}\|u\|_{L^{\frac{20}{7}}}^{2} L^{\frac{20}{7}}(Q(\rho))
\end{aligned}
$$


With the help of the triangle inequality, interpolation inequality (2.7) and the last inequality, we get

$$
\begin{aligned}
\|u\|_{L^{\frac{20}{7}, \frac{15}{4}}(Q(r))}^{2} \leq & \|v\|_{L^{\frac{20}{7}, \frac{15}{4}}(Q(r))}^{2}+\left\|\nabla \Pi_{h}\right\|_{L^{\frac{20}{7}, \frac{15}{4}}(Q(r))}^{2} \\
\leq & C\left\{\|v\|_{L^{2, \infty}(Q(r))}^{2}+\|\nabla v\|_{L^{2}(Q(r))}^{2}\right\}+\frac{r^{\frac{8}{5}}}{(\rho-r)^{\frac{21}{10}}}\|u\|_{L^{\frac{20}{7}} L^{\frac{20}{7}}(Q(\rho))}^{2} \\
\leq & \left\{\frac{C}{(\rho-r)^{2}}+\frac{C \rho^{\frac{21}{10}}}{(\rho-r)^{\frac{41}{10}}}\right\}\|u\|_{L^{20 / 7}\left(Q\left(\frac{r+\rho}{2}\right)\right)}^{4} \\
& +\left\{\frac{C \rho^{3 / 2}}{(\rho-r)^{2}}+\frac{C r^{\frac{8}{5}}}{(\rho-r)^{\frac{21}{10}}}\right\}\|u\|_{L^{20 / 7}(Q(\rho))}^{2}+\frac{1}{16}\|\nabla u\|_{L^{2}(Q(\rho))}^{2} .
\end{aligned}
$$

Employing (2.5) and (2.3) once again, we have the estimate

$$
\left\|\nabla^{2} \Pi_{h}\right\|_{L^{2}(Q(r))}^{2} \leq \frac{C r^{3}}{(\rho-r)^{3+2 \cdot 1}}\left\|\nabla \Pi_{h}\right\|_{L^{2}\left(Q\left(\frac{r+\rho}{2}\right)\right)}^{2} \leq \frac{C r^{3} \rho^{3 / 2}}{(\rho-r)^{3+2 \cdot 1}}\|u\|_{L^{20 / 7}(Q(\rho))}^{2} .
$$

This together with the triangle inequality and (3.11) leads to

$$
\begin{aligned}
\|\nabla u\|_{L^{2}(Q(r))}^{2} & \leq\|\nabla v\|_{L^{2}(Q(r))}^{2}+\left\|\nabla^{2} \Pi_{h}\right\|_{L^{2}(Q(r))}^{2} \\
\leq & \left\{\frac{C}{(\rho-r)^{2}}+\frac{C \rho^{\frac{21}{10}}}{(\rho-r)^{\frac{41}{10}}}\right\}\|u\|_{L^{20 / 7}(Q(\rho))}^{4} \\
& +\left\{\frac{C \rho^{3 / 2}}{(\rho-r)^{2}}+\frac{C r^{3} \rho^{3 / 2}}{(\rho-r)^{3+2 \cdot 1}}\right\}\|u\|_{L^{20 / 7}(Q(\rho))}^{2}+\frac{1}{16}\|\nabla u\|_{L^{2}(Q(\rho))}^{2} .
\end{aligned}
$$

Eventually, we infer that

$$
\begin{aligned}
& \|u\|_{L^{\frac{20}{7}, \frac{15}{4}}(Q(r))}^{2}+\|\nabla u\|_{L^{2}(Q(r))}^{2} \\
\leq & \left\{\frac{C}{(\rho-r)^{2}}+\frac{C \rho^{\frac{21}{10}}}{(\rho-r)^{\frac{41}{10}}}\right\}\|u\|_{L^{20 / 7}(Q(\rho))}^{4} \\
& +\left\{\frac{C \rho^{3 / 2}}{(\rho-r)^{2}}+\frac{C r^{3} \rho^{3 / 2}}{(\rho-r)^{3+2 \cdot 1}}+\frac{C r^{\frac{8}{5}}}{(\rho-r)^{\frac{21}{10}}}\right\}\|u\|_{L^{20 / 7}(Q(\rho))}^{2}+\frac{3}{16}\|\nabla u\|_{L^{2}(Q(\rho))}^{2} .
\end{aligned}
$$

Now, we are in a position to apply lemma 2.2 to the latter to find that

$$
\|u\|_{L^{\frac{20}{T}}, \frac{15}{4}\left(Q\left(\frac{R}{2}\right)\right)}^{2}+\|\nabla u\|_{L^{2}\left(Q\left(\frac{R}{2}\right)\right)}^{2} \leq C R^{-\frac{1}{2}}\|\nabla u\|_{L^{\frac{20}{7}}(Q(R))}^{2}+C R^{-2}\|\nabla u\|_{L^{\frac{20}{T}}(Q(R))}^{4} .
$$

This achieves the proof of this proposition.

\section{Induction arguments and proof of Theorem 1.2}

In this section, we begin with a critical proposition, which can be seen as the bridge between

the previous step and the next step for the given statement in the induction arguments. Ultimately, we finish the proof of Theorem 1.2. 
Proposition 4.1. Assume that $\iint_{Q(r)}|v|^{\frac{10}{3}} \leq r^{5} N$ with $r_{k} \leq r \leq r_{k_{0}}$. There is a constant $C$ such that the following result holds. For any given $\left(x_{0}, t_{0}\right) \in \mathbb{R}^{n} \times \mathbb{R}^{-}$and $k_{0} \in \mathbb{N}$, we have for any $k>k_{0}$,

$$
\begin{aligned}
& \sup _{-r_{k}^{2} \leq t-t_{0} \leq 0} f_{\tilde{B}_{k}}|v|^{2}+r_{k}^{-3} \iint_{\tilde{Q}_{k}}|\nabla v|^{2} \\
& \leq C \sup _{-r_{k_{0}}^{2} \leq t-t_{0} \leq 0} f_{\tilde{B}_{k_{0}}}|v|^{2}+C \sum_{l=k_{0}}^{k} r_{l}\left(f \int_{\tilde{Q}_{l}}|v|^{\frac{10}{3}}\right)^{\frac{9}{10}}+C \sum_{l=k_{0}}^{k} r_{l}^{\frac{3}{10}}\left(\iint_{\tilde{Q}_{l}}|v|^{\frac{10}{3}}\right)^{\frac{3}{5}}\left(\iint_{Q_{1}}|u|^{\frac{20}{7}}\right)^{\frac{7}{20}} \\
& +C \sum_{l=k_{0}}^{k} r_{l}^{\frac{13}{10}}\left(f \int_{\tilde{Q}_{l}}|v|^{\frac{10}{3}}\right)^{\frac{3}{5}}\left(\iint_{Q_{1}}|u|^{\frac{20}{7}}\right)^{\frac{7}{20}}+C \sum_{l=k_{0}}^{k} r_{l}^{\frac{3}{5}}\left(\iint_{\tilde{Q}_{l}}|v|^{\frac{10}{3}}\right)^{\frac{3}{10}}\left(\iint_{Q_{1}}|u|^{\frac{20}{7}}\right)^{\frac{7}{10}} \\
& +C \sum_{l=k_{0}}^{k} r_{l}\left(\iint_{\tilde{Q}_{l}}|v|^{\frac{10}{3}}\right)^{\frac{3}{10}}\left(\iint_{\tilde{Q}_{1}}|\nabla u|^{2}\right)^{\frac{1}{2}}+C \sum_{l=k_{0}}^{k} r_{l}^{\frac{3}{2}}\left(\iint_{\tilde{Q}_{l}}|v|^{\frac{10}{3}}\right)^{\frac{3}{10}}\left\{N^{3 / 5}\right. \\
& \left.+N^{\frac{3}{10}}\left(\iint_{Q_{1}}|u|^{\frac{20}{7}}\right)^{\frac{7}{20}}+\left(\iint_{Q_{1}}|u|^{20 / 7}\right)^{\frac{7}{10}}\right\} .
\end{aligned}
$$

Proof. Without loss of generality, we suppose $\left(x_{0}, t_{0}\right)=(0,0)$. We denote the backward heat kernel

$$
\Gamma(x, t)=\frac{1}{4 \pi\left(r_{k}^{2}-t\right)^{3 / 2}} e^{-\frac{|x|^{2}}{4\left(r_{k}^{2}-t\right)}} .
$$

In addition, consider the smooth cut-off functions below

$$
\phi(x, t)= \begin{cases}1, & (x, t) \in Q\left(r_{k_{0}+1}\right), \\ 0, & (x, t) \in Q^{c}\left(\frac{3}{2} r_{k_{0}+1}\right)\end{cases}
$$

satisfying

$$
0 \leq \phi, \phi_{2} \leq 1 \text { and } r_{k_{0}}^{2}\left|\partial_{t} \phi(x, t)\right|+r_{k_{0}}^{l}\left|\partial_{x}^{l} \phi(x, t)\right| \leq C .
$$

To proceed further, we list some properties of the test function $\phi(x, t) \Gamma(x, t)$, whose deduction rests on elementary calculations.

(i) There is a constant $c>0$ independent of $r_{k}$ such that, for any $(x, t) \in Q\left(r_{k}\right)$,

$$
\Gamma(x, t) \geq c r_{k}^{-3} \text {. }
$$

(ii) For any $(x, t) \in Q\left(r_{k_{0}}\right)$, we have

$$
|\Gamma(x, t) \phi(x, t)| \leq C r_{k}^{-3}, \quad|\nabla \phi(x, t) \Gamma(x, t)| \leq C r_{k}^{-4}, \quad|\phi(x, t) \nabla \Gamma(x, t)| \leq C r_{k}^{-4} .
$$

(iii) For any $(x, t) \in Q\left(3 r_{k_{0}} / 4\right) \backslash Q\left(r_{k_{0}} / 2\right)$, one can deduce that

$$
\Gamma(x, t) \leq C r_{k_{0}}^{-3}, \partial_{i} \Gamma(x, t) \leq C r_{k_{0}}^{-4},
$$

which yields that

$$
\left|\Gamma(x, t) \partial_{t} \psi(x, t)\right|+|\Gamma(x, t) \Delta \psi(x, t)|+|\nabla \psi(x, t) \nabla \Gamma(x, t)| \leq C r_{k_{0}}^{-5} .
$$


(iv) For any $(x, t) \in Q_{l} \backslash Q_{l+1}$,

$$
\Gamma \leq C r_{l+1}^{-3}, \nabla \Gamma \leq C r_{l+1}^{-4}
$$

Now, setting $\varphi_{1}=\phi \Gamma$ in the local energy inequality (1.8) and utilizing the fact that $\Gamma_{t}+\Delta \Gamma=0$, we see that

$$
\begin{aligned}
& \int_{B_{1}}|v|^{2} \phi(x, t) \Gamma+\int_{-r_{k_{0}}^{2}}^{t} \int_{B_{1}}|\nabla v|^{2} \phi(x, s) \Gamma \\
\leq & \int_{-r_{k_{0}}^{2}}^{t} \int_{B_{1}}|v|^{2}\left(\Gamma \Delta \phi+\Gamma \partial_{t} \phi+2 \nabla \Gamma \nabla \phi\right) \\
& +\int_{-r_{k_{0}}^{2}}^{t} \int_{B_{1}}|v|^{2} v \cdot \nabla(\phi \Gamma)-|v|^{2} \nabla \Pi_{h} \cdot \nabla \phi \\
& +\int_{-r_{k_{0}}^{2}}^{t} \int_{B_{1}} \Gamma \phi\left(v \otimes v-v \otimes \nabla \Pi_{h}: \nabla^{2} \Pi_{h}\right)+\int_{-r_{k_{0}}^{2}}^{t} \int_{B_{1}} \Pi_{1} v \cdot \nabla(\Gamma \phi)+\int_{-r_{k_{0}}^{2}}^{t} \int_{B_{1}} \Pi_{2} v \cdot \nabla(\Gamma \phi)
\end{aligned}
$$

where

$$
\nabla \Pi_{1}=\mathcal{W}_{2, B_{1}}(\Delta u), \quad \nabla \Pi_{2}=-\mathcal{W}_{\frac{20}{7}, B_{1}}(\nabla \cdot(u \otimes u))
$$

First, we present the low bound estimates of the terms on the left hand side of this inequality. Indeed, with the help of (iv), we find

$$
\int_{B_{k}}|v|^{2} \phi \Gamma \geq C f_{B_{k}}|v|^{2}
$$

and

$$
\int_{-r_{k_{0}}^{2}}^{t} \int_{B_{1}} \phi \Gamma|\nabla v|^{2} \geq r_{k}^{-n} \iint_{Q_{k}}|\nabla v|^{2}
$$

Having observed that the support of $\partial_{t} \phi$ is included in $\tilde{Q}\left(\frac{3 r_{k_{0}}}{4}\right) / \tilde{Q}\left(\frac{r_{k_{0}}}{2}\right)$, we get

$$
\int_{-r_{k_{0}}^{2}}^{t} \int_{B_{1}}|v|^{2}\left|\Gamma \Delta \phi+\Gamma \partial_{t} \phi+2 \nabla \Gamma \nabla \phi\right| \leq C \sup _{-r_{k_{0}}^{2} \leq t \leq 0} f_{B_{k_{0}}}|v|^{2} .
$$

Hölder's inequality and (iv) enable us to write that

$$
\begin{aligned}
& \iint_{Q_{k_{0}}}|v|^{2} v \cdot \nabla(\phi \Gamma) d \tau \\
\leq & \sum_{l=k_{0}}^{k-1} \iint_{Q_{l} / Q_{l+1}}|v|^{3}|\nabla(\phi \Gamma)|+\iint_{Q_{k}}|v|^{3}|\nabla(\phi \Gamma)| \\
\leq & \sum_{l=k_{0}}^{k} r_{l}^{-4} \iint_{Q_{l}}|v|^{3} \\
\leq & C \sum_{l=k_{0}}^{k} r_{l}\left(\int_{Q_{l}}|v|^{\frac{10}{3}}\right)^{\frac{9}{10}} .
\end{aligned}
$$


Following the lines of reasoning which led to the last inequality, we have

$$
\begin{aligned}
& \iint_{Q_{k_{0}}}|v|^{2} \nabla \Pi_{h} \cdot \nabla(\phi \Gamma) \\
\leq & \sum_{l=k_{0}}^{k} r_{l}^{-4} \iint_{Q_{l}}|v|^{2}\left|\nabla \Pi_{h}\right| \\
\leq & C \sum_{l=k_{0}}^{k} r_{l}^{\frac{3}{10}}\left(\iint_{Q_{l}}|v|^{\frac{10}{3}}\right)^{\frac{3}{5}}\left(\iint_{Q_{1}}|u|^{\frac{20}{7}}\right)^{\frac{7}{20}} .
\end{aligned}
$$

Likewise, we have

$$
\begin{aligned}
& \iint_{Q_{k_{0}}}|v|^{2}\left|\nabla^{2} \Pi_{h}\right|(\phi \Gamma) \\
\leq & \sum_{l=k_{0}}^{k} r_{l}^{-3} \iint_{Q_{l}}|v|^{2}\left|\nabla^{2} \Pi_{h}\right| \\
\leq & C \sum_{l=k_{0}}^{k} r_{l}^{\frac{13}{10}}\left(\iint_{Q_{l}}|v|^{\frac{10}{3}}\right)^{\frac{3}{5}}\left(\iint_{Q_{1}}|u|^{\frac{20}{7}}\right)^{\frac{7}{20}} .
\end{aligned}
$$

Using Hölder's inequality again, (iv), (2.5) and (2.3), we infer that

$$
\begin{aligned}
& \iint_{Q_{k_{0}}} \phi \Gamma|v|\left|\nabla \Pi_{h}\right|\left|\nabla^{2} \Pi_{h}\right| \\
\leq & C \sum_{l=k_{0}}^{k} r_{l}^{-n}\left(\iint_{Q_{l}}|v|^{\frac{10}{3}}\right)^{\frac{3}{10}}\left(\iint_{Q_{l}}\left|\nabla \Pi_{h}\right|^{\frac{20}{7}}\right)^{\frac{7}{20}}\left(\iint_{Q_{l}}\left|\nabla^{2} \Pi_{h}\right|^{\frac{20}{7}}\right)^{\frac{7}{20}} \\
\leq & C \sum_{l=k_{0}}^{k} r_{l}^{\frac{3}{5}}\left(\iint_{Q_{l}}|v|^{\frac{10}{3}}\right)^{\frac{3}{10}}\left(\iint_{Q_{1}}|u|^{\frac{20}{7}}\right)^{\frac{7}{10}} .
\end{aligned}
$$

Set $\chi_{l}=1$ on $|x| \leq 7 / 8 r_{l}$ and $\chi_{l}=0$ if $|x| \geq r_{l} . \quad \chi_{k_{0}} \Gamma=\Gamma$ on $Q_{k_{0}}$ By the support of $\left(\chi_{l}-\chi_{l+1}\right)$, we derive from (iv) that $\left|\nabla\left(\left(\chi_{l}-\chi_{l+1}\right) \phi \Gamma\right)\right| \leq C r_{l+1}^{-4}$. With the help of (iv) again, we see that $\left|\nabla\left(\chi_{k} \phi \Gamma\right)\right| \leq C r_{k}^{-4}$. Therefore, it holds

$$
\begin{aligned}
\iint_{Q_{k_{0}}} v \cdot \nabla(\phi \Gamma) \Pi_{1} & =\sum_{l=k_{0}}^{k-1} \iint_{Q_{l}} v \cdot \nabla\left(\left(\chi_{l}-\chi_{l+1}\right) \phi \Gamma\right) \Pi_{1}+\iint_{Q_{k}} v \cdot \nabla\left(\chi_{k} \phi \Gamma\right) \Pi_{1} \\
& =\sum_{l=k_{0}}^{k-1} \iint_{Q_{l}} v \cdot \nabla\left(\left(\chi_{l}-\chi_{l+1}\right) \phi \Gamma\right)\left(\Pi_{1}-\overline{\Pi_{1 l}}\right)+\iint_{Q_{k}} u \cdot \nabla\left(\chi_{k} \phi \Gamma\right)\left(\Pi_{1}-\bar{\Pi}_{1 k}\right) \\
& \leq C \sum_{l=k_{0}}^{k-1} r_{l+1}^{-4} \iint_{Q_{l}}|v|\left|\Pi_{1}-\bar{\Pi}_{1 l}\right|+r_{k}^{-4} \iint_{Q_{k}}|v|\left|\Pi_{1}-\bar{\Pi}_{1 k}\right| \\
& =: I+I I .
\end{aligned}
$$

The Hölder inequality, (2.6) and (2.4) give

$$
I \leq C \sum_{l=k_{0}}^{k-1} r_{l+1}^{-4}\left(\iint_{\tilde{Q}_{l}}|v|^{\frac{10}{3}}\right)^{\frac{3}{10}}\left(\iint_{\tilde{Q}_{l}}\left|\Pi_{1}-\overline{\Pi_{1 l}}\right|^{2}\right)^{\frac{1}{2}} r_{l}
$$




$$
\begin{aligned}
& \leq C \sum_{l=k_{0}}^{k-1} r_{l+1}^{-\frac{5}{2}}\left(\iint_{Q_{l}}|v|^{\frac{10}{3}}\right)^{\frac{3}{10}} r_{l}^{\frac{7}{2}}\left(\iint_{\tilde{Q}_{1}}\left|\Pi_{1}-\overline{\Pi_{11}}\right|^{2}\right)^{\frac{1}{2}} \\
& \leq C \sum_{l=k_{0}}^{k-1} r_{l+1}\left(\iint_{Q_{l}}|v|^{\frac{10}{3}}\right)^{\frac{3}{10}}\left(\iint_{\tilde{Q}_{1}}\left|\Pi_{1}\right|^{2}\right)^{\frac{1}{2}} \\
& \leq C \sum_{l=k_{0}}^{k-1} r_{l+1}\left(\iint_{Q_{l}}|v|^{\frac{10}{3}}\right)^{\frac{3}{10}}\left(\iint_{\tilde{Q}_{1}}|\nabla u|^{2}\right)^{\frac{1}{2}}
\end{aligned}
$$

and

$$
I I \leq C r_{k}\left(\iint_{Q_{l}}|v|^{\frac{10}{3}}\right)^{\frac{3}{10}}\left(\iint_{\tilde{Q}_{1}}|\nabla u|^{2}\right)^{\frac{1}{2}}
$$

which turns out that

$$
\iint_{\tilde{Q}_{k_{0}}} v \cdot \nabla(\phi \Gamma) \Pi_{1} \leq C \sum_{l=k_{0}}^{k} r_{l}\left(\iint_{Q_{l}}|v|^{\frac{10}{3}}\right)^{\frac{3}{10}}\left(\iint_{\tilde{Q}_{1}}|\nabla u|^{2}\right)^{\frac{1}{2}} .
$$

Note that

$$
u \otimes u=v \otimes v-v \otimes \nabla \Pi_{h}-\nabla \Pi_{h} \otimes v+\nabla \Pi_{h} \otimes \nabla \Pi_{h} .
$$

For $r_{k} \leq r \leq r_{k_{0}}$, we compute directly that

$$
\iint_{Q(r)}\left|v \otimes v-\overline{v \otimes v_{l}}\right|^{10 / 7} \leq \iint_{Q(r)}|v|^{20 / 7} \leq C r^{5}\left(\iint_{Q(r)}|v|^{\frac{10}{3}}\right)^{6 / 7} \leq C r^{5} N^{6 / 7} .
$$

The Hölder inequality and (2.5) ensure that

$$
\begin{aligned}
\iint_{Q(r)}\left|v \otimes \nabla \Pi_{h}-\overline{v \otimes \nabla \Pi_{h l}}\right|^{10 / 7} & \leq C \iint_{Q(r)}\left|v \otimes \nabla \Pi_{h}\right|^{10 / 7} \\
& \leq C\left(\iint|v|^{\frac{10}{3}}\right)^{\frac{3}{7}}\left(\iint\left|\nabla \Pi_{h}\right|^{\frac{20}{7}}\right)^{\frac{1}{2}} r^{\frac{5}{14}} \\
& \leq C r^{4}\left(\iint_{Q(r)}|v|^{\frac{10}{3}}\right)^{\frac{3}{7}}\left(\iint_{Q_{1}}|u|^{\frac{20}{7}}\right)^{\frac{1}{2}} \\
& \leq C r^{4} N^{\frac{3}{7}}\left(\iint_{Q_{1}}|u|^{\frac{20}{7}}\right)^{\frac{1}{2}} .
\end{aligned}
$$

In view of Poincaré inequality for a ball, Hölder's inequality, (2.5) and (2.3), we arrive at

$$
\begin{aligned}
& \iint_{Q(r)}\left|\nabla \Pi_{h} \otimes \nabla \Pi_{h}-\overline{\nabla \Pi_{h} \otimes \nabla \Pi_{h l}}\right|^{10 / 7} \\
\leq & C r^{10 / 7}\left(\iint_{Q(r)}\left|\nabla \Pi_{h}\right|^{20 / 7}\right)^{\frac{1}{2}}\left(\iint_{Q(r)}\left|\nabla^{2} \Pi_{h}\right|^{20 / 7}\right)^{\frac{1}{2}} \\
\leq & C r^{\frac{31}{7}}\left(\iint_{Q_{1}}\left|\nabla \Pi_{h}\right|^{20 / 7}\right)^{\frac{1}{2}}\left(\iint_{Q_{1}}\left|\nabla \Pi_{h}\right|^{20 / 7}\right)^{\frac{1}{2}} \\
\leq & C r^{\frac{31}{7}}\left(\iint_{Q_{1}}|u|^{20 / 7}\right) .
\end{aligned}
$$

We deduce from (4.4)-(4.7) that

$$
\iint_{Q(r)}\left|u \otimes u-\overline{(u \otimes u)_{r}}\right|^{10 / 7} \leq C r^{5} N^{6 / 7}+r^{4} N^{\frac{3}{7}}\left(\iint_{Q_{1}}|u|^{\frac{20}{7}}\right)^{\frac{1}{2}}+C r^{\frac{31}{7}}\left(\iint_{Q_{1}}|u|^{20 / 7}\right)
$$




$$
\leq C r^{4}\left\{N^{6 / 7}+N^{\frac{3}{7}}\left(\iint_{Q_{1}}|u|^{\frac{20}{7}}\right)^{\frac{1}{2}}+C\left(\iint_{Q_{1}}|u|^{20 / 7}\right)\right\}
$$

With (4.8) in hand, we can apply Lemma 2.3 to obtain that

$$
\iint_{Q(r)}\left|\Pi_{2}-\overline{\Pi_{2}} B(r)\right|^{10 / 7} \leq C r^{4}\left\{N^{6 / 7}+N^{\frac{3}{7}}\left(\iint_{Q_{1}}|u|^{\frac{20}{7}}\right)^{\frac{1}{2}}+C\left(\iint_{Q_{1}}|u|^{20 / 7}\right)\right\} .
$$

Particulary, for any $k \leq l \leq k_{0}$, it holds

$$
\iint_{Q_{l}}\left|\Pi_{2}-\bar{\Pi}_{2} B_{l}\right|^{10 / 7} \leq C r_{l}^{4}\left\{N^{6 / 7}+N^{\frac{3}{7}}\left(\iint_{Q_{1}}|u|^{\frac{20}{7}}\right)^{\frac{1}{2}}+C\left(\iint_{Q_{1}}|u|^{20 / 7}\right)\right\} .
$$

By the Hölder inequality, we see that

$$
r_{l}^{-4} \iint_{Q_{l}}|v|\left|\Pi_{2}-\bar{\Pi}_{2} B(r)\right| \leq C r_{l}^{-4}\left(\iint_{\tilde{Q}_{l}}|v|^{\frac{10}{3}}\right)^{\frac{3}{10}}\left(\iint_{\tilde{Q}_{l}}\left|\Pi_{2}-{\overline{\left(\Pi_{2}\right)}}_{B_{l}}\right|^{\frac{10}{7}}\right)^{\frac{7}{10}} .
$$

Plugging (4.9) into (4.11), we have

$$
\begin{aligned}
& \iint_{Q_{k_{0}}} v \cdot \nabla(\phi \Gamma) \Pi_{2} \\
\leq & C \sum_{l=k_{0}}^{k-1} r_{l+1}^{-4} \iint_{Q_{l}}|v|\left|\Pi_{2}-\overline{\Pi_{2 l}}\right|+r_{k}^{-4} \iint_{Q_{k}}|v|\left|\Pi_{2}-\overline{\Pi_{2 k}}\right| \\
\leq & C \sum_{l=k_{0}}^{k} r_{l}^{\frac{3}{10}}\left(\iint_{Q_{l}}|v|^{\frac{10}{3}}\right)^{\frac{3}{10}}\left\{N^{6 / 7}+N^{\frac{3}{7}}\left(\iint_{Q_{1}}|u|^{\frac{20}{7}}\right)^{\frac{1}{2}}+C\left(\iint_{Q_{1}}|u|^{20 / 7}\right)\right\}^{\frac{7}{10}} .
\end{aligned}
$$

Finally, collected these estimates leads to (4.17).

With Proposition 4.1 at our disposal, we will now present the proof of Theorem 1.1 .

Proof of Theorem 1.2. By the interior estimate (2.5) of harmonic function and (3.2), we have

$$
\left\|\nabla \Pi_{h}\right\|_{L^{\infty}(\tilde{B}(1 / 8))} \leq C\left\|\nabla \Pi_{h}\right\|_{L^{20 / 7}(B(1))} \leq C\|u\|_{L^{20 / 7}(B(1))} .
$$

Assume for a while we have proved that, for any Lebesgue point $\left(x_{0}, t_{0}\right) \in Q(1 / 8)$,

$$
\left|v\left(x_{0}, t_{0}\right)\right| \leq C \text {. }
$$

We derive from (4.12) and (4.13) that

$$
\|u\|_{L^{20 / 7, \infty}(\tilde{Q}(1 / 8))} \leq\left\|\nabla \Pi_{h}\right\|_{L^{20 / 7, \infty}(\tilde{Q}(1 / 8))}+\|v\|_{L^{20 / 7, \infty}(\tilde{Q}(1 / 8))} \leq C\|u\|_{L^{20 / 7}(Q(1))} .
$$

By the well-known Serrin regularity criteria in [12], we know that $(0,0)$ is a regular point. Therefore, it remains to prove (4.13). In what follows, let $\left(x_{0}, t_{0}\right) \in Q(1 / 8)$ and $r_{k}=2^{-k}$. According to the Lebesgue differentiation theorem, it suffices to show

$$
\int_{\tilde{Q}_{k}}|v|^{\frac{10}{3}} \leq \varepsilon_{1}^{2 / 3}, \quad k \geq 3
$$


First, we show that (4.14) is valid for $k=3$. Indeed, from (3.11) in section 3, Proposition 3.1 and hypothesis 1.12, we know that

$$
\begin{aligned}
& \sup _{-\left(\frac{3}{8}\right)^{2} \leq t \leq 0} \int_{B\left(\frac{3}{8}\right)}|v|^{2} d x+\iint_{Q\left(\frac{3}{8}\right)}|\nabla v|^{2} d x d \tau \\
& \leq C\|u\|_{L^{20 / 7}\left(Q\left(\frac{1}{2}\right)\right)}^{4}+C\|u\|_{L^{20 / 7}\left(Q\left(\frac{1}{2}\right)\right)}^{2}+\frac{1}{16}\|\nabla u\|_{L^{2}\left(Q\left(\frac{1}{2}\right)\right)}^{2}, \\
& \leq C \varepsilon^{7 / 10} .
\end{aligned}
$$

In light of Sobolev embeddings and the Young inequality, we see that

$$
\left(\iint_{Q\left(\frac{3}{8}\right)}|v|^{\frac{10}{3}}\right)^{\frac{3}{10}} \leq C\left(\sup _{-\left(\frac{3}{8}\right)^{2} \leq t<0} \int_{B\left(\frac{3}{8}\right)}|v|^{2}\right)^{1 / 2}+C\left(\iint_{Q\left(\frac{3}{8}\right)}|\nabla v|^{2}\right)^{1 / 2} .
$$

It turns out that

$$
\iint_{\tilde{Q}_{3}}|v|^{\frac{10}{3}} \leq C \varepsilon_{1}^{\frac{7}{6}}
$$

This proves (4.14) in the case $k=3$. Now, we assume that, for any $3 \leq l \leq k$,

$$
\iint_{\tilde{Q}_{l}}|v|^{\frac{10}{3}} \leq \varepsilon_{1}^{2 / 3}
$$

Furthermore, there holds, for any $r_{k} \leq r \leq r_{3}$

$$
\iint_{\tilde{Q}(r)}|v|^{\frac{10}{3}} \leq C \varepsilon_{1}^{2 / 3}
$$

For any $3 \leq i \leq k$, by Proposition 4.1 with $N=C \varepsilon_{1}^{2 / 3}$, (1.12) and the above induction hypothesis, we find that

$$
\begin{aligned}
& \sup _{-r_{k}^{2} \leq t-t_{0} \leq 0} f_{\tilde{B}_{k}}|v|^{2}+r_{k}^{-n} \iint_{\tilde{Q}_{k}}|\nabla v|^{2} \\
& \leq C \sup _{-r_{3}^{2} \leq t-t_{0} \leq 0} f_{\tilde{B}_{k_{0}}}|v|^{2}+C \sum_{l=k_{0}}^{k} r_{l}\left(\iint_{Q_{l}}|v|^{\frac{10}{3}}\right)^{\frac{9}{10}} \\
& +C \sum_{l=3}^{k} r_{l}^{\frac{3}{10}}\left(f \int_{Q_{l}}|v|^{\frac{10}{3}}\right)^{\frac{3}{5}}\left(\iint_{Q_{1}}|u|^{\frac{20}{7}}\right)^{\frac{7}{20}} \\
& +C \sum_{l=3}^{k} r_{l}^{\frac{13}{10}}\left(\iint_{Q_{l}}|v|^{\frac{10}{3}}\right)^{\frac{3}{5}}\left(\iint_{Q_{1}}|u|^{\frac{20}{7}}\right)^{\frac{7}{20}} \\
& +C \sum_{l=3}^{k} r_{l}^{\frac{3}{5}}\left(\iint_{Q_{l}}|v|^{\frac{10}{3}}\right)^{\frac{3}{10}}\left(\iint_{Q_{1}}|u|^{\frac{20}{7}}\right)^{\frac{7}{10}} \\
& +C \sum_{l=3}^{k} r_{l}\left(\iint_{Q_{l}}|v|^{\frac{10}{3}}\right)^{\frac{3}{10}}\left(\iint_{\tilde{Q}_{1}}|\nabla u|^{2}\right)^{\frac{1}{2}} \\
& +C \sum_{l=3}^{k} r_{l}^{\frac{3}{10}}\left(\iint_{Q_{l}}|v|^{\frac{10}{3}}\right)^{\frac{3}{10}}\left\{N^{3 / 5}+N N^{\frac{3}{10}}\left(\iint_{Q_{1}}|u|^{\frac{20}{7}}\right)^{\frac{7}{20}}+\left(\iint_{Q_{1}}|u|^{20 / 7}\right)^{\frac{7}{10}}\right\}
\end{aligned}
$$




$$
\begin{aligned}
\leq & C \varepsilon^{7 / 5}+C \sum_{l=3}^{k} r_{l} \varepsilon^{\frac{3}{5}}+C \sum_{l=3}^{k} r_{l}^{\frac{3}{10}} \varepsilon^{\frac{2}{5}} \varepsilon^{\frac{7}{20}}+C \sum_{l=3}^{k} r_{l}^{\frac{13}{10}} \varepsilon^{\frac{2}{5}} \varepsilon^{\frac{7}{20}} \\
& +C \sum_{l=3}^{k} r_{l}^{\frac{3}{5}} \varepsilon^{\frac{1}{5}} \varepsilon^{\frac{7}{10}}+C \sum_{l=3}^{k} r_{l} \varepsilon^{\frac{1}{5}} \varepsilon^{\frac{7}{20}}+C \sum_{l=3}^{k} r_{l}^{\frac{3}{10}} \varepsilon^{\frac{1}{5}}\left\{\varepsilon^{\frac{2}{5}}+\varepsilon^{\frac{1}{5}} \varepsilon^{\frac{7}{20}}+\varepsilon^{\frac{7}{10}}\right\} \\
\leq & C \varepsilon^{\frac{11}{20}} .
\end{aligned}
$$

Invoking the Gagliardo-Nirenberg inequality, we deduce that

$$
\int_{\tilde{B}_{k+1}}|v|^{\frac{10}{3}} d x \leq C\left(\int_{\tilde{B}_{k}}|v|^{2}\right)^{\frac{2}{3}}\left[\left(\int_{\tilde{B}_{k}}|\nabla v|^{2}\right)^{1 / 2}+r_{k}^{-1}\left(\int_{\tilde{B}_{k}}|v|^{2}\right)^{1 / 2}\right]^{2},
$$

which means

$$
\iint_{\tilde{Q}_{k+1}}|v|^{\frac{10}{3}} \leq C\left(\sup _{-r_{k}^{2} \leq t-t_{0}<0} \int_{\tilde{B}_{k}}|v|^{2}\right)^{\frac{2}{3}}\left(\iint_{\tilde{Q}_{k}}|\nabla v|^{2}\right)+\left(\sup _{-r_{k}^{2} \leq t-t_{0}<0} \int_{\tilde{B}_{k}}|v|^{2}\right)^{5 / 3} .
$$

This inequality, combined with (4.17), implies that

$$
\begin{aligned}
\frac{1}{r_{k+1}^{5}} \iint_{\tilde{Q}_{k+1}}|v|^{\frac{10}{3}} \leq & C\left(\frac{1}{r_{k}^{3}} \sup _{-r_{k}^{2} \leq t-t_{0}<0} \int_{\tilde{B}_{k}}|v|^{2}\right)^{\frac{5}{3}} \\
& +C\left(\frac{1}{r_{k}^{3}} \sup _{-r_{k}^{2} \leq t-t_{0}<0} \int_{\tilde{B}_{k}}|v|^{2}\right)^{\frac{3}{2}}\left(r_{k}^{-3} \iint_{\tilde{Q}_{k}}|\nabla v|^{2}\right) \\
\leq & C \varepsilon_{1}^{\frac{11}{12}} .
\end{aligned}
$$

Collecting the above bounds, we eventually conclude that

$$
\iint_{\tilde{Q}_{k+1}}|v|^{\frac{10}{3}} \leq \varepsilon_{1}^{2 / 3}
$$

This completes the proof of this theorem.

\section{Acknowledgement}

Jiu was partially supported by the National Natural Science Foundation of China (No.11671273, No.11231006). The research of Wang was partially supported by the National Natural Science Foundation of China under grant No. 11601492 and the the Youth Core Teachers Foundation of Zhengzhou University of Light Industry. The research of Zhou is supported in part by the National Natural Science Foundation of China under grant No. 11401176 and Doctor Fund of Henan Polytechnic University (No. B2012-110).

\section{References}

[1] L. Caffarelli, R. Kohn and L. Nirenberg, Partial regularity of suitable weak solutions of the Navier-Stokes equations, Comm. Pure. Appl. Math., 35 (1982), 771-831. 
[2] D. Chae and J. Wolf, Removing discretely self-similar singularities for the 3D NavierStokes equations. Comm. Partial Differential Equations. 42 (2017), 1359-1374.

[3] _ On the Liouville type theorems for self-similar solutions to the Navier-Stokes equations. Arch. Ration. Mech. Anal. 225 (2017), 549-572.

[4] G. Galdi, C. Simader, and H. Sohr, On the Stokes problem in Lipschitz domains, Annali di Mat. pura ed appl. (IV), 167 (1994), pp. 147-163.

[5] M. Giaquinta, Multiple integrals in the calculus of variations and nonlinear elliptic systems. Annals of Mathematics Studies, 105. Princeton University Press, Princeton, NJ, 1983.

[6] C. Guevara and N. C. Phuc, Local energy bounds and $\varepsilon$-regularity criteria for the 3D Navier-Stokes system. Calc. Var., (2017) 56:68.

[7] I. Kukavica, Regularity for the Navier-Stokes equations with a solution in a Morrey space. Indiana Univ. Math. J. 57 (2008), 2843-2860.

[8] O. Ladyzenskaja and G. Seregin, On partial regularity of suitable weak solutions to the three-dimensional Navier-Stokes equations, J. Math. Fluid Mech., 1 (1999), 356-387.

[9] F. Lin, A new proof of the Caffarelli-Kohn-Nirenberg Theorem, Comm. Pure Appl. Math., 51 (1998), 241-257.

[10] C. He, Y. Wang and D. Guo, New $\varepsilon$-regularity criteria and application to the box dimension of the singular set in the 3D Navier-Stokes equations, arxiv: 1709.01382.

[11] W. Ren, Y. Wang and G. Wu, Gang, Partial regularity of suitable weak solutions to the multi-dimensional generalized magnetohydrodynamics equations. Commun. Contemp. Math., 18 (2016), 1650018, 38 pp.

[12] J. Serrin, On the interior regularity of weak solutions of the Navier-Stokes equations, Arch. Rational Mech. Anal., 9 (1962), 187-195.

[13] M. Struwe, Partial regularity results for the Navier-Stokes equations, Comm. Pure Appl. Math., , 41 (1988), 437-458.

[14] L. Tang and Y. Yu, Partial regularity of suitable weak solutions to the fractional Navier-Stokes equations, Comm. Math. Phys. 334 (2015) 1455-1482.

[15] Wolf, J.: A new criterion for partial regularity of suitable weak solutions to the Navier-Stokes equations. Adv. Math. Fluid Mech.. Rannacher, A.S.R. ed., pp. 613630. Springer (2010)

[16] Wolf, J.: On the local regularity of suitable weak solutions to the generalized NavierStokes equations. Ann. Univ. Ferrara, 61 (2015), 149-171. 